\title{
GLASGOW UNIVERSITY RADIOCARBON MEASUREMENTS V
}

\author{
M. ERGIN,* D. D. HARKNESS,** and A. WALTON† \\ Chemistry Department, The University, Glasgow, W. 2
}

\section{INTRODUCTION}

Operation of counting systems and preparation of results remain as described previously by Baxter et al. (1969), and Ergin et al. (1970).

\section{ACKNOWLEDGMENTS}

Financial support for the reported studies was provided by the Medical Research Council and the Natural Environment Research Council. Thanks are due to numerous organizations and individuals throughout the world who have assisted us in the supply of suitable samples. Our gratitude is again extended to the National Physical Laboratory for their continued provision of facilities for $\mathrm{C}^{13} / \mathrm{C}^{12}$ measurement.

\section{SAMPLE DESCRIPTIONS}

\section{ATMOSPHERIC $\mathrm{CO}_{2}$ SAMPLES}

\section{A. Global distribution}

Data relate to research program of transport of $\mathrm{C}^{14}$ within the "dynamic" carbon reservoir (Walton et al., 1970). $\mathrm{CO}_{2}$ coll. by exposure of carbonate-free $8 \mathrm{M} \mathrm{KOH}$ solution to atmosphere at ground level for each calendar month.

\section{Lerwick, Scotland series}

Samples coll. by Meteorologic Office in their ventilated East hut, Lerwick $\left(60^{\circ} 08^{\prime} \mathrm{N} \mathrm{Lat,} 01^{\circ} 11^{\prime} \mathrm{W}\right.$ Long).

\section{Lerwick series, 1969}

\begin{tabular}{lcccc}
\hline Sample no. & Coll. date & $\delta \mathrm{C}^{14} \%$ & $\delta \mathrm{C}^{13} \% / \%$ & $\Delta \%$ \\
\hline GU-334 & Jan. & $54.6 \pm 2.1$ & -20.6 & $53.2 \pm 2.2$ \\
GU-335 & May & $55.8 \pm 2.0$ & -22.7 & $55.1 \pm 2.0$ \\
GU-336 & July & $56.9 \pm 2.2$ & -18.5 & $54.9 \pm 2.0$ \\
GU-337 & Oct. & $53.1 \pm 2.0$ & -19.6 & $51.4 \pm 2.0$ \\
\hline
\end{tabular}

\section{Gibraltar series}

Samples coll. by Meteorologic Office, R.A.F., Gibraltar, in well ventilated room, adjacent to open window $\left(36^{\circ} 09^{\prime}\right.$ Lat, $05^{\circ} 21^{\prime} \mathrm{W}$ Long).

\footnotetext{
* Hacettepe University, Ankara, Turkey.

** $\mathrm{C}^{14}$ Laboratory, Scottish Rescarch Reactor Centre, East Kilbride, Scotland. Cianada.

† Atlantic Oceanographic Laboratory, Bedford Institute, Dartmouth, Nova Scotia,
} 
Gibraltar series, 1969

\begin{tabular}{lcccc}
\hline Sample no. & Coll. date & $\delta \mathrm{C}^{14 \%}$ & $\delta \mathrm{C}^{13 \%} \%$ & $\Delta \%$ \\
\hline GU-338 & Jan. & $52.9 \pm 2.2$ & -19.2 & $51.1 \pm 2.2$ \\
GU-339 & April & $55.5 \pm 2.2$ & -21.4 & $54.4 \pm 2.3$ \\
GU-340 & July & $54.8 \pm 2.1$ & -22.2 & $53.9 \pm 2.1$ \\
GU-341 & Oct. & $52.6 \pm 1.5$ & -21.8 & $51.6 \pm 1.5$ \\
\hline
\end{tabular}

\section{Hong Kong series}

Samples coll. by Meteorologic Office at Tates Cairn radar sta. in Stevenson screen which shelters samples from both rain and dry deposition $\left(22^{\circ} 18^{\prime} \mathrm{N}\right.$ Lat, $14^{\circ} 10^{\prime} \mathrm{E}$ Long).

\section{Hong Kong series, 1969}

\begin{tabular}{lcccc}
\hline Sample no. & Coll. date & $\delta \mathrm{C}^{140} \%$ & $\delta \mathrm{C}^{13 \%} \%$ & $\Delta \%$ \\
\hline GU-342 & Jan. & $79.6 \pm 2.5$ & -20.2 & $77.8 \pm 2.5$ \\
GU-343 & April & $48.8 \pm 2.2$ & -24.2 & $48.6 \pm 2.2$ \\
GU-344 & July & $46.8 \pm 2.0$ & -23.4 & $46.3 \pm 2.0$ \\
GU-345 & Oct. & $53.8 \pm 2.3$ & -21.0 & $52.6 \pm 2.4$ \\
\hline
\end{tabular}

\section{Pretoria series}

Samples coll. by Atomic Energy Board, Pelindaba, Pretoria, in Stevenson screen housing a variety of meteorologic instruments $\left(25^{\circ} 45^{\prime}\right.$ $S$ Lat, $28^{\circ} 16^{\prime}$ E Long).

\section{Pretoria series, 1969}

\begin{tabular}{lcccc}
\hline Sample no. & Coll. date & $\delta \mathrm{C}^{14 \%} \%$ & $\delta \mathrm{C}^{13 \%} \%$ & $\Delta \%$ \\
\hline GU-346 & Jan. & $52.2 \pm 2.2$ & -23.7 & $51.8 \pm 2.2$ \\
GU-347 & April & $48.8 \pm 2.1$ & -24.5 & $48.7 \pm 2.1$ \\
GU-348 & July & $49.9 \pm 2.2$ & -22.0 & $49.0 \pm 2.2$ \\
GU-349 & Oct. & $49.3 \pm 2.2$ & -23.2 & $48.8 \pm 2.2$ \\
\hline
\end{tabular}

\section{Stanley, Falkland Islands series}

Samples coll. outdoors by Meteorologic Office, Stanley, Falkland Is., in meteorologic thermometer screen ( $51^{\circ} 42^{\prime} \mathrm{S}$ Lat, $57^{\circ} 52^{\prime} \mathrm{W}$ Long). 
Stanley series, 1969

\begin{tabular}{lcccc}
\hline Sample no. & Coll. date & $\delta \mathrm{C}^{14} \%$ & $\delta \mathrm{C}^{13 \%} \%$ & $\Delta \%$ \\
\hline GU-350 & Jan. & $51.1 \pm 2.2$ & -24.7 & $51.0 \pm 2.2$ \\
GU-351 & April & $49.7 \pm 2.5$ & -24.9 & $49.7 \pm 2.5$ \\
GU-352 & July & $50.2 \pm 1.5$ & -23.5 & $49.7 \pm 1.5$ \\
GU-353 & Oct. & $46.6 \pm 2.0$ & -24.5 & $46.5 \pm 2.0$ \\
\hline
\end{tabular}

\section{B. Urban variations}

The reported $\mathrm{C}^{14}$ activities were measured during a study of combustion product $\mathrm{CO}_{2}$ levels in urban air viz., local Suess effect (Walker, 1969).

Samples were coll. during Jan. 1969 through exposure of $8 \mathrm{M} \mathrm{KOH}$ at selected sites within a 30 -mi. radius of Glasgow, Scotland $\left(55^{\circ} 50^{\prime} \mathrm{N}\right.$ Lat, $04^{\circ} 16^{\prime} \mathrm{W}$ Long).

\section{Urban $\mathrm{CO}_{2}$, Glasgow area}

\begin{tabular}{crlccc}
\hline $\begin{array}{c}\text { Sample } \\
\text { no. }\end{array}$ & $\begin{array}{c}\text { Coll. } \\
\text { site } \\
\text { no. }\end{array}$ & District & $\delta \mathrm{C}^{1+\%} \%$ & $\delta \mathrm{C}^{13 \%} \%$ & $\Delta \%$ \\
\hline GU-354 & 1 & Beith & $50.9 \pm 1.1$ & -23.2 & $50.3 \pm 1.2$ \\
GU-355 & 2 & Mauchline & $49.7 \pm 1.0$ & -23.0 & $49.1 \pm 1.1$ \\
GU-356 & 3 & Mauchline & $46.3 \pm 0.5$ & -24.4 & $46.2 \pm 0.6$ \\
GU-357 & 4 & Newton Mearns & $47.9 \pm 1.0$ & -23.9 & $47.6 \pm 1.1$ \\
GU-358 & 5 & Newton Mearns & $47.1 \pm 0.9$ & -24.5 & $46.9 \pm 1.0$ \\
GU-359 & 6 & Newton Mearns & $41.6 \pm 0.8$ & -23.7 & $41.2 \pm 0.9$ \\
GU-360 & 7 & Central Glasgow & $41.4 \pm 0.9$ & -23.2 & $40.9 \pm 1.0$ \\
GU-361 & 8 & Central Glasgow & $40.4 \pm 0.9$ & -23.9 & $39.9 \pm 1.0$ \\
GU-362 & 9 & Central Glasgow & $39.4 \pm 1.1$ & -23.0 & $38.9 \pm 1.2$ \\
GU-363 & 10 & Riddrie & $38.6 \pm 1.1$ & -21.7 & $37.7 \pm 1.2$ \\
GU-364 & 11 & Riddrie & $23.1 \pm 0.9$ & -23.5 & $22.7 \pm 1.0$ \\
GU-365 & 12 & Wishaw & $27.5 \pm 0.9$ & -23.4 & $27.1 \pm 1.0$ \\
\hline Comman
\end{tabular}

Comment: data correlate with geographic distribution of industry and prevailing wind pattern. The importance of careful site selection in global $\mathrm{C}^{14}$ studies is emphasized by up to ca. $29 \%$ excess 'fossil' $\mathrm{CO}_{2}$ in air at certain locations.

\section{SOIL CARBON}

Data relate to profile coll. from non-calcareous, imperfectly drained Brown Forest soil belonging to the Lanfine Association. Samples coll. Jan. 1970 in vicinity of Doonbank Farm, Ayr, Scotland (55 $28^{\prime} \mathrm{N}$ Lat, 04 $38^{\prime}$ W Long). Natl. Grid Ref. NS 327184.

Samples were sieved ( $1 \mathrm{~mm}$ mesh) and washed several times in $2 \mathrm{M}$ HCl to remove organic debris (Gunning, 1970). 
Doonbank Farm series

\begin{tabular}{|c|c|c|c|c|c|}
\hline $\begin{array}{l}\text { Sample } \\
\text { no. }\end{array}$ & Soil fraction & Coll. depth & $\delta \mathrm{C}^{14 \%} \%$ & $\delta \mathrm{C}^{13} \%$ & $\Delta \%$ \\
\hline$\overline{\text { GU-366 }}$ & $\begin{array}{l}\text { Alkali sol. } \\
\text { carbon }\end{array}$ & $0-3 \mathrm{~cm}$ & $-0.3 \pm 0.5$ & -31.4 & $1.0 \pm 0.7$ \\
\hline GU-367 & $\begin{array}{l}\text { Alkali insol. } \\
\text { carbon }\end{array}$ & $0-3 \mathrm{~cm}$ & $-0.6 \pm 0.6$ & -30.5 & $0.5 \pm 0.8$ \\
\hline GU & $\mathrm{Tc}$ & $0-3$ & $7.6 \pm 0.7$ & -29.0 & $8.4 \pm 0.8$ \\
\hline G & To & $8-10$ & -4.4 & -30.5 & $-3.4 \pm 0.8$ \\
\hline GU-3' & Total carbon & $13-15 \mathrm{~cm}$ & -12.8 & -29.5 & $-12.1 \pm 0.9$ \\
\hline GU-371 & Total carbon & $23-25 \mathrm{~cm}$ & -24.3 & -27.8 & $-23.6 \pm 0.8$ \\
\hline GU-372 & Total carbon & $40-45 \mathrm{~cm}$ & $-32.9 \pm 0.6$ & -28.8 & $-32.4 \pm 0.8$ \\
\hline
\end{tabular}

Comment: presence of 'bomb' $\mathrm{C}^{14}$ is evident in all surface samples, although enrichment is small. Temporal variations of such $\mathrm{C}^{14}$ activities may afford a measure of the rates of mineralization and transport of organic carbon in soils.

III. GEOLOGIC SAMPLES

$8950 \pm 90$

GU-373. Dundonald Burn, Irvine

7000 B.C.

Organic mud exposed in bank of Dundonald Burn, $\left(55^{\circ} 36^{\prime} \mathrm{N}\right.$ Lat, $04^{\circ} 38^{\prime} \mathrm{W}$ Long), Natl. Grid Ref. NS 337372, $290 \mathrm{~m}$ N of Shewalton Bridge, near Irvine, Ayrshire, Scotland. Sample is top $5 \mathrm{~cm}$ of organic mud ca. $30 \mathrm{~cm}$ thick, overlain by sand, and underlain by gravelly till. Top of organic mud is at alt $6.2 \mathrm{~m}$ (Newlyn). Coll. 1966 and subm. by W. G. Jardine, Dept. Geol., Univ. Glasgow. Comment (W. G. J.): date is a more accurate maximum for beginning of main Flandrian marine transgression in central Ayrshire than date for wood from near middle or base of same bed of organic mud (Q-642: $9575 \pm 150$, Godwin and Willis, 1962).

\section{GU-374. Hollanbank Cottage}

Marine shells (Cardium sp.) from emerged shell ridge $50 \mathrm{~m} \mathrm{~N}$ of Hollanbank Cottage, Kirkcudbrightshire, Scotland, (54 $52^{\circ} \mathrm{N}$ Lat, $04^{\circ}$ 22' W Long), Natl. Grid Ref. NX 482555. From alt. $5.24 \mathrm{~m}$ (Newlyn) 46 $\mathrm{cm}$ below top of shell ridge. Coll. 1966 and subm. by W. G. Jardine. Comment (W.G.J.): date indicates time of shell-ridge formation. It is close to age of shell layer within laminated fine sand at similar alt. at Crook of Baldon on W side of Wigtown Bay (I-5068: $2290 \pm 95$, in press).

GU-375. Newbie Mains Borehole

Organic mud from undisturbed sample from borehole $500 \mathrm{~m}$ NNW of Newbie Mains Farm, Dumfriesshire, Scotland, $\left(54^{\circ} 58^{\prime} \mathrm{N}\right.$ Lat, $03^{\circ} 17^{\prime}$ W Long), Natl. Grid Ref. NY 171651. Organic mud, $10 \mathrm{~cm}$ thick, occurs 
within marine fine sand/sand sequence. Top of organic mud at alt. 4.57 m (Newlyn). Coll. 1967 and subm. by W. G. Jardine. Comment (W.G.J.): date is consistent with others for organic deposits assoc. with Carse deposits in E part of Solway Firth area (Q-637: $8135 \pm 150$, Godwin and Willis, 1962; GU-64: $7254 \pm 101$, Baxter et al., 1969). Supports suggestion that penetration of kettles in this area by Flandrian transgressive sea was diachronous (Jardine, in press).

\section{REFERENCES}

Baxter, M. S., Ergin, M., and Walton, A., 1969, Glasgow University radiocarbon measurements I: Radiocarbon, v. 11, p. 43-52.

Ergin, M., Harkness, D. D., and Walton, A., 1970, Glasgow University radiocarbon measurements II: Radiocarbon, v. 12, p. 486-495.

Godwin, H. and Willis, E. H., 1962, Cambridge University natural radiocarbon measurements V: Radiocarbon, v. 4, p. 57-70.

Gunning, D., 1970, B.Sc. thesis, Univ. of Glasgow.

Jardine, W. G., Form and age of late Quaternary shorelines and coastal deposits of south-west Scotland: Critical date: Quaternaria, in press.

Walker, S., 1969, B.Sc. thesis, Univ. of Glasgow.

Walton, A., Ergin, M., and Harkness, D. D., 1970, Carbon-14 concentrations in the atmosphere and carbon dioxide exchange rates: Jour. Geophys. Research, v. 75, p. $3089-3098$. 